\title{
The question on origin of the universe and Big Bang
}

\author{
Krishna Raj Adhikari \\ Paschimanchal Campus, IOE (TU), Pokhara, Nepal \\ kra534812@yahoo.com
}

\begin{abstract}
School of thought is the theory of creation (theism) and school of thought deals with the random chance of evolution (atheism) about the origin of the universe and origin of the life. In the race of proof of the hypothesis, the theism has no scientific evidence and reliable proof, on the other hand atheism based on the scientific observable evidence. The latest theory of origin of the universe by Big Bang is more believable and supported by some scientific evidence such as Doppler effect on light, Hubble observation and result of the expanding the universe and observation of the cosmic microwave background radiation(CMBR). Paper briefly discussing about the origin of the universe and the Bing Bang.
\end{abstract}

\section{Introduction}

We are living on the earth which is a part of solar system where it is a member of Milky Way galaxy and the galaxy is the major component of the universe. The galaxies, nebulae, cosmic microwave background radiation, dark-matter etc constitute the universe. Now questions arise, when and where did the universe and our earth originate? When I was a young boy around 10 years, answers of every difficult task and question converged either to the king or to the god/divine because of our strong believe, at that time, onto the king and the unseen god to solve the unsolved problems and mysteries. Now, here is a long debate about the existence of the god and the answer of the question of origin of the universe, our earth and origin of life on the planet. Here, about these topics our human society is divided into two major categories in each case.

\section{Hypothesis and their development}

Based upon the ideas and arguments of the philosophers, religions and scientists here are two major schools of thought about the origin of the universe, the earth and the life on it. According to the first category of thought (theism) the universe was created by a well skilled and intelligent creator called god/divine. The universe was designed, created and operated as His will and creative ability. It does not require any evidence and proof, only believe and trust upon god is "sufficient." The second school of thought is that the universe was not created by the Wise Creator rather it was evolving by the random chance and afterwards every thing and event is governed by the laws of science. The saying of the S.W.
Hawking about this question is that "The problems of the origin of the universe, is a bit the old question: which came first, the chicken or the egg. In other words what agency created the universe? And what created the agency or perhaps, the universe, or the agency that created it, existed forever, and did not need to be created". Yes, when and where did the creator create the universe? Where the creator did make the plan, design, and create the whole things? They do not have the satisfactory answer. Only the matter is that the trust/believe upon the god is sufficient and we must trust that all these phenomena are as per His will.

If the god or divine was the true creator of the universe and its components than we must have to thank the god for His perfect, absolute and valuable creation where every essential thing is created and necessary methods are suggested to run the universe and the life. But there are no strong and persuasive evidences to trust upon this "perfect creation". Actually, without reservation, everything and natural phenomena in this universe is governed by the laws of science but the thing is that some unsolved problems are still remaining as the mysteries; they are also governed by the unpredicted/predicted laws. Thus, in human society, the question on origin of the universe is either the creation was made by the Wise Creator (theism) or the random chance of evolution of the universe (atheism).

Christians, Islamic religions' and western traditions predicted that the universe was created by the divine in the fairly recent past about six thousands years ago. On the other hand the Hindu religion and 
the eastern philosophy believe that the universe was created about 2860000000 ( $=2.86$ billion) years ago which is nearly the age of the origin of the life. But scientific technique found that the earth was evolved $4540000000(=4.54$ billion $)$ years ago to the present day, is far away from the estimated age suggested by religion. The opinion or version about the creation of the universe (theism) also does not match with each other because they are just logics and philosophy which are not based upon the scientific evidence.

Aristotle the Greek philosopher believed that "the universe had existed and would exist forever" but he did not believe "the universe had a beginning". Another philosopher Immanuel Kant, in 1781, wrote a monumental where he concluded that there were equally valid arguments, both for believing that the universe had a beginning, and for believing that it did not ( $W$ Hawking: origin of the universe, $p p 1)$. Any way all these ideas pledged that the universe is steady and unchanging in time either it had been created in its present form or it had existed forever, like it is today. Even, Newton and Einstein (he added so called "cosmological constant") also pledged the static model of the universe. Later, Einstein said: the cosmological constant was the greatest mistake of my life(S W Hawking: origin of the universe, $p p 2)$.

Doppler's effect for light and observations result of Edwin Hubble in the 1920's, was that the distant galaxies were moving away from us where the speed of recession was roughly proportional to their distance from us. That is, the distance between the galaxies is increasing with time and now we can say, the universe is not static or the universe is expanding. This evidence, after the Hubble observation, demands a complete change in the previous concepts on the origin of the universe.

\section{The new look: Big Bang}

The Hubble observations announced the universe is expanding (i.e., the distance between the galaxies is increasing), if so when we reverse the time and motion of the universe back then the galaxies approaching each other and ultimately they should all have been piled up and concentrated into a very dense hot-point having infinite space-time curvature and temperature at some moment between ten to twenty billion years ago. This hot point, early universe, at which the universe were concentrated and contracted, is known as Primordial fire ball. It was exploded at about 13.7 billion years ago which is called Big Bang under such conditions all the known laws of science would break down. After this event the fragments of the primordial fire ball had been shattered in all directions and got cooled down. The Big Bang is now considered as the origin/ beginning of the universe and the time. The explanation on the origin of stars, galaxies and planet, based on the theory of Big Bang, are briefly discussed below.

After $10^{-43}$ second of Big Bang the temperature of the universe was about $10^{32}$ Kelvin, average energy per particle was $10^{19} \mathrm{GeV}$ and that time was the era of the theory of every thing (ToE).Roughly the time $10^{-42}$ to $10^{-35}$ second after the big explosion, the universe was like a ball of very hot soup of the quarks and leptons (electron, neutrino, muon and tau) where strong and electroweak forces were unified and the particles were being transferred into each other. As we know, every particle has antiparticle such as: protonantiproton, electron-positron, neutrino-antineutrino etc. During the period of unification of strong-electroweak forces (GUT's period) there were creation and annihilation of the particles and anti-particles to release the energy as photons where the baryon number was not conserved. Thus, by the end of this period the number of quarks has been greater than the anti-quarks. During the time $10^{-35}$ to $10^{-6}$, second the galaxies were recessed with a high speed, became less dense and a result it cooled off and the universe was composed of the mixture of quarks, leptons and mediator particles such as gluons, photon, weak bosons $\mathrm{w}^{+}$and $\mathrm{z}^{0}$. At the time $10^{-6} \mathrm{~s}$ the universe cooled to $10^{13} \mathrm{~K}$ and energy of per particle was $1 \mathrm{GeV}$, and quarks and anti-quarks were united by the strong forces to form protons, neutrons (both collectively called nucleons) and their anti-particles. Again there were formation and annihilation of the nucleon and anti-nucleon with the release of tremendous radiation (energy) which resulted into slightly more nucleons than anti-nucleons. As photon energy fell well below the limiting value for the pair production, at 14 second, there was only the annihilation process in action; as a result there were more particles than the anti-particles of each.

At the beginning of the period $1 \mathrm{~s}$ to $225 \mathrm{~s}$ the temperature was about $10^{10} \mathrm{~K}$ which is favorable condition for conversion of free neutron (with mean life $887 \mathrm{~s}$ ) into proton, beta $(\beta)$, and anti neutrino as a result neutron-proton ratio got decreased; and this process continued till 225s. But at the end of this period, the universe had 
been cooled down enough, so the circumstance was favorable to collide and stick the proton and neutron by strong nuclear forces to form nuclei of low mass number deuteron $\left(\mathrm{H}^{2}\right)$ which can absorb neutron to form trion $\left(\mathrm{H}^{3}\right)$ and it can absorb proton to form $\mathrm{He}^{3}$ which is unstable and which can again absorb a neutron to form helium nucleus $\left(\mathrm{He}^{4}\right) . \mathrm{He}^{3}$ and $\mathrm{He}^{4}$ combine (fuse) to form lithium nucleus $\left(\mathrm{Li}^{7}\right)$. Such a process of formation of nuclei at favorable condition by the combination of protons and neutrons also some times even by the fusion of lighter nuclei is called nucleosynthesis. During the fusion process there was decreased in mass which is eventually transformed into energy as photons according to the Einstein's mass-energy equivalence relation $\mathrm{E}=\mathrm{mc}^{2}$, where $\mathrm{m}$ is decreased in mass during fusion and $\mathrm{c}$ is velocity of light in vacuum. Thus, the universe had plenty of radiation at that time but the light could not travel through because of opaque nature of hot soup of lighter nuclei. At the time $10^{13}$ s (about 0.380 million years) the temperature of the universe was about $3000 \mathrm{~K}$ where electrostatics force came into play to attract the positively charged nucleus and negatively charged electron to form neutral hydrogen and helium atoms. These neutral atoms got pulled closer together by the gravitational force of attraction to form clouds of gas and finally the stars and cluster of stars called galaxy. In a star, at the sufficient condition, the ionized hydrogen atoms under went fusion to form helium nucleus $\left(\mathrm{He}^{4}\right)$ and released sufficient energy, and developed a large temperature, which is one of the sources of the light and heat energy (radiation) of the star. This time the universe was transparent because the neutral atoms could not absorb the radiations or photons, so, the radiations left over at that time on all parts and in all directions of the universe is observable now a days as cosmic microwave background radiation (CBMR).

When the star uses up its hydrogen, inward gravitational pressure becomes greater than outward radiation and gaseous pressure, so the core of star starts to contract which results into an increase of kinetic energy of the atoms in the star. For the star of the adequate mass having sufficient energy and density, two helium nuclei fuse to form beryllium nucleus $\left(\mathrm{Be}^{8}\right)$, which is unstable so, it fuses with $\mathrm{He}^{4}$ to form $\mathrm{C}^{12}$ nucleus. The process continues to form $\mathrm{O}^{16}$, $\mathrm{Ne}^{20}$ and $\mathrm{Mg}^{24}$ nuclei. Again $\mathrm{C}^{12}$ and $\mathrm{O}^{16}$ unite/fuse to form heavier nuclei and in some cases the final product is the stable $\mathrm{Fe}^{56}$ nucleus. The nucleosynthesis process was continuing to form heaver nuclei with the release of tremendous energy so that the massive star may finally explode as supernova. This explosion sent out the heavier elements, radiations and other debris into the space. This debris got united again by the gravitational attraction to form second generation stars and planets, asteroids etc. Our sun, falls into this category, and the solar system were originated after the explosion of the supernova.

\section{Origin of the life}

Again, about the question on the origin of life on the earth, there are also two schools of thought. One aspect is the creation of the life by Wise Creator, Brahma, according to the Hindu religion (theism), which created, supported and ruled the universe and the living being to come up into present status. Another school of thought proposed that as the planets were evolved and cooled gradually down to a favorable condition to synthesize the inorganic matters to form amino acids, protein and eventually the simple form of the life, which was really a random chance of evolution of the life. The simple form of life was progressively evolving / changing into the diversity of life existing at present days by the effects of the environment, place and availability of food which were explained well by Darwin in his popular theory Darwinism as theory of natural selection and survival of fittest. The same condition does not exist to evolve the simple form of life from the inorganic matters, so, we now can not see /observe the formation of the life from the inorganic matters.

\section{Conclusion}

I mentioned, in earlier discussion that, there are two schools of thought on the origin of the universe and origin of the life. In terms of first thought i.e., theism, they put forwarded their opinions, logics, hypothesis, and even long and interesting stories to hold up their hypothesis of the "divine theory of creation". They do not think to require any scientific observations and evidence because the trust /believe upon the unseen god, who had designed and created the things that are present today, is "sufficient to prove their theory". But on the other hand the theory of evolution (atheism) is based on some scientific observations and evidences such as Doppler's effect for light, Hubble observations and conclusion on expanding the universe, but do think that our earth is expanding, and the observations of cosmic microwave background radiation detected/notified by the radio-astronomers Arno Penzias and

Robert Wilson in 1964. In the actual sense these two observations 
are the great evidence or landmark test of the Big Bang model of origin of the universe. In 1985 Georges Lemaitre, a Belgian physicist remarked at a scientific meeting with strong believes on the Big Bang as: It is as certain the universe started with a Big Bang about 15 billon years ago as it is that the earth goes round the sun. All the evidences are not the proof themselves but we know that the evidences help to come up into a conclusion. Thus, the other theories and hypotheses about the origin of the universe and the origin of the life are the wishful logics and philosophy without strong evidence and scientific observations where as the Big Bang is based on the scientific observations and evidence so it is most believable theory known so far. So the universe was originated / evolved with a Big Bang and count down of the time was also begun with that Big Bang. There was no space and time before Big Bang because there was no universe!

\section{References}

1. Halliday D; 1997. Robert Resnic and Jearl Walker Fundamental of physics extended fifth edition, John Willey and Sons.Ins.

2. http;/en.wikepedia.org/wiki/cosmic_microwave_ background_radiation.

3. http;/en.wikepedia.org/wiki/origin_of_the_universe.

4. Hugh D. young and Roger A. freedman, Sears and Zemansky's University of physics with modern physics, $12^{\text {th }}$ edition, Pearson education.

5. Stephen w. Hawking, 1988. the text of origin of the universe.

6. Stephen w. Hawking, 1996. The brief history of time, the updated and expanded tenth anniversary edition, Bantam Book.

7. Stephen w. Hawking \& Leonard Mlodinow, 2010. The Grand Design, Bantam Press. 